\title{
Chikungunya-an emerging infection in Bangladesh and role of physiotherapist for recovering patients
}

\section{Editorial}

Chikungunya fever is a mosquito-borne illness of humans caused by the chikungunya virus, a type of alphavirus. The virus was first reported in 1952 in Tanzania. The disease is not confined to Africa now a day rather it is being out breaking in the South-East Asia Region. Due to its complex nature in progress, number of Chikungunya fever and arthritis patients increased among the inhabitants of Dhaka city. One online newspaper of Bangladesh published that 196 patients with Chikungunya was identified between May to June 2017 in Institute of Epidemiology, Disease Control and Research (IEDCR) and 45 infected patients were also found at Bangabandhu Sheikh Mujib Medical University (BSMMU).

The incubation period after being bitten by an infected mosquito ranges from 2-12 days, most commonly between 3-7 days. The disease may evolve in three phases: acute or febrile (lasting up to 10 days), sub-acute (11-90days) and chronic ( $>90$ days). Approximately $50 \%$ of people who experience acute infection develop chronic joint pain that can last from months to years. Besides, most patients recover fully. Symptoms characteristic of an acute infection includes biphasic high fever lasting from a few days to few weeks. Back pain, fatigue, myalgia and arthralgia may or may not present with joint swelling. World Health Organization (WHO) guideline found fever $(92 \%)$ usually associated with arthralgia $(87 \%)$ backache $(67 \%)$ and headache $(62 \%)$ among patients. Experimental models of arthritis induced by viruses suggest that different cytokines, chemokines and other inflammatory mediators are produced; these are related to the intensity of inflammation in the acute phase. They also involved in the recruitment of macrophages and $\mathrm{T}$ lymphocytes to the site of viral replication and cause dysregulation of inflammation in this stage leading to the expression of other inflammatory proteins responsible for damaging the joint in the chronic phase. Despite of its pathogenesis in chemical in nature, mechanical problems such atrophy in muscle, decrease aerobic capacity, restricted joint range of motion exaggerated by pain and prolong immobility following subside of acute phase of fever. WHO recommended for Physiotherapy to minimize joint pain and disability after completion of acute phase of the disease.

The treatment protocol for recovering patients was based on WHO guideline. During the first week, ice compression for 10 minutes for

\author{
Volume 2 Issue I - 2017 \\ Mohammad Habibur Rahman \\ Department of Physiotherapy, Bangladesh Health Professions \\ Institute, Bangladesh
}

Correspondence: Mohammad Habibur Rahman, Assistant Professor, Department of Physiotherapy, BHPI, CRP, Chapain, Savar, Dhaka-I343, Bangladesh, Email sumonpt1983@gmail.com

Received:September 12, 2017 | Published: November 0I, 2017

2- 3hourly, active assisted movement within the pain free range as per patient tolerance, non-weight bearing exercise especially in the lower limb joints can be applied. In the second week pulley assisted exercises, isometric exercise and close kinetic chain exercises using self-body weight as per patient tolerance can be prescribed. In the third week of treatment, patients can continue mild form of aerobic and stretching exercise. In addition, patients also can perform home exercise program consisting of active exercises of upper and lower limb joints, low effect aerobic exercise such as walking in the corridor for five minutes with comfortable pace, static stretching exercise of major muscle groups and hold for 10 second hold in each muscles. Patient can carry out home exercise program 2 sessions daily for 20 minutes in each session. Chikungunya fever not only increased high grade fever but also create severe disability because of joint pain and subsequent immobility. Pain is the most common clinical manifestation of Chikungunya, is difficult to control, compromising the quality of life of affected patients. Hence, dose based physiotherapy can helps in recovering from joint pain and residual disability.

\section{Acknowledgements}

None.

\section{Conflict of interest}

The author declares no conflict of interest. 